\title{
EFFICIENT SIMULATION OF CRACK PROPAGATION IN COMPLEX GEOMETRIES USING A PHASE-FIELD MODEL AND THE FINITE CELL METHOD
}

\author{
L. Hug ${ }^{\dagger *}$, S. Kollmannsberger ${ }^{\dagger}$, J. Kirschke ${ }^{\ddagger}$, M. Potten $"$, G. Stockinger", K. Thuro", \\ Z. Yosibash ${ }^{\S}$ and E. Rank ${ }^{\dagger}$ \\ ${ }^{\dagger}$ Chair of Computational Modeling and Simulation, Technical University of Munich, \\ Arcisstr. 21, 80333 Munich, Germany \\ \$ Department of Neuroradiology, Technical University of Munich, \\ Ismaninger Str. 22, 81675 Munich, Germany \\ "Chair of Engineering Geology, Technical University of Munich, \\ Arcisstr. 21, 80333 Munich, Germany
}

$\S$ School of Mechanical Engineering, The Iby and Aladar Fleischman Faculty of Engineering, Tel-Aviv University, Ramat-Aviv 69978, Israel

*E-mail: lisa.hug@tum.de,web page: https://www.cms.bgu.tum.de/en/

\begin{abstract}
Phase-field models for fracture are based on a diffused approximation of the crack using a scalar field variable, which altogether avoids the modeling of discontinuities. Although widely used and extended to a broad range of applications, phase-field simulations of large-scale scenarios featuring complex geometries and crack patterns are still a challenging task. In this contribution, a numerical framework is proposed which combines a phase-field model for brittle fracture with an adaptive refinement technique and an embedded domain approach to an efficient tool for the simulation of complex, 3-dimensional crack scenarios.
\end{abstract}

In the phase-field approach, the regularization of the sharp crack is based on a length-scale parameter which governs the extent of diffusion and determines the numerical width of the crack. For small length-scale parameters, a very fine mesh is needed at least in the vicinity of the crack to fully resolve the resulting crack profile. As this can quickly become expensive, Nagaraja et al. [3] combined multi-level hp-refinement [4] and a phase-field approach for brittle fracture in two dimensions to allow for a locally refined mesh which dynamically adapts to the crack path. In a second step, the Finite Cell Method (FCM) [1] was successfully integrated into the model to enable the efficient simulation of complex geometries. The numerical framework was extended to $3 \mathrm{D}$ and validated in terms of crack propagation and nucleation in homogeneous and isotropic material based on a newly suggested benchmark problem [2].

To demonstrate the potential of the proposed numerical framework, two application examples of complex and heterogeneous geometries based on CT-scan data are considered. In a first application, compression fracture of human vertebrae is simulated numerically and validity of the phase-field model is discussed in a comparison with experimental data. In a second part, uniaxial compression tests on geological core samples are simulated. Both examples demonstrate the advantages of combining a phase-field model with the FCM and multi-level hp-refinement for the efficient simulation of complex crack propagation phenomena. 


\section{REFERENCES}

[1] Düster, Alexander, et al. "The finite cell method for three-dimensional problems of solid mechanics." Computer methods in applied mechanics and engineering 197.45-48 (2008): 37683782 .

[2] Hug, Lisa, et al. "A 3D benchmark problem for crack propagation in brittle fracture." Computer Methods in Applied Mechanics and Engineering 364 (2020): 112905.

[3] Nagaraja, Sindhu, et al. "Phase-field modeling of brittle fracture with multi-level hp-FEM and the finite cell method." Computational Mechanics 63.6 (2019): 1283-1300.

[4] Zander, Nils, et al. "Multi-level hp-adaptivity: high-order mesh adaptivity without the difficulties of constraining hanging nodes." Computational Mechanics 55.3 (2015): 499-517.
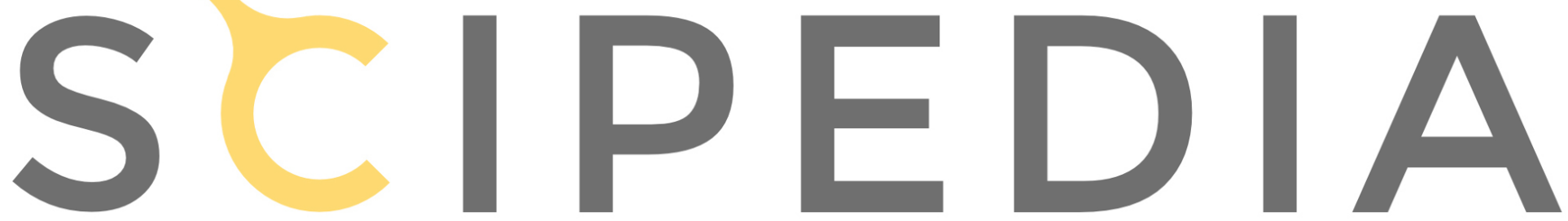